\title{
ON THE APPLICATION OF A THETA FORMULA TO REPRESENTATION IN BINARY QUADRATIC FORMS*
}

\author{
BY GORDON PALL $†$
}

In his remarkable 1885 dissertation, $\ddagger$ Applications of the Theory of Elliptic Functions to the Theory of Numbers, Nazimoff uses the formula (1) to derive the number of representations in $x^{2}+3 y^{2}$ and $x^{2}+5 y^{2}$. Some of his theorems are not quite correct. He does not derive the series representing $\sum \sum q^{x^{2}+7 y^{2}}$ since "the derivation is long and not difficult." He states that the method has only limited application, since generally in (4) the $A$ 's are functions of $k$.

It is the purpose of this paper to give the essential details for $x^{2}+7 y^{2}$, and to prove that the only cases $a x^{2}+b y^{2}, a b$ odd, which are actually solvable by Nazimoff's method are $a=1, b=1,3$, 5,7 . The final result for $x^{2}+7 y^{2}$ is of course far from new.

At the time this paper was written the writer intended to examine the products of series involved in other cases than $a=1, b=1,3,5,7$, in the hope of obtaining information on $N\left(n=x^{2}+11 y^{2}\right)$ or other cases of several classes in a genus. But since then an arithmetic method has been discovered of finding a simple formula for the number of representations in any positive, binary quadratic form. It is possible, after the details of this theory have been worked out, that there may be applications to elliptic and modular functions.

The formula which Nazimoff uses is

$$
\sum_{x, y} q^{a x^{2}+b y^{2}}=(a b)^{-1 / 2} \phi(2 K /(a b)),
$$

where $x, y$ run through all integers, $a$ and $b$ are given positive, odd, relative-prime in tegers, and

$$
\phi(u)=\frac{2 K}{\pi} \prod_{l=1}^{(a-1) / 2} t\left(l b v_{i}\right\rangle \prod_{h=1}^{(b-1) / 2} t(h a u) .
$$

* Presented to the Society at Pasadena, November 28, 1931.

$\dagger$ National Research Fellow, California Institute of Technology.

$\ddagger$ Translation by Arnold E. Ross, pp. 5-12. 
Here $t(u)$ is an abbreviation for the elliptic function

$$
\frac{\operatorname{sn} u \operatorname{dn} u}{\operatorname{cn} u}=\frac{\vartheta_{1}(x) \vartheta_{3}(x)}{\vartheta_{2}(x) \vartheta_{4}(x)}, \quad \quad(u=2 K x) .
$$

Thus $N\left(n=a x^{2}+b y^{2}\right)$ is the coefficient of $q^{n}$ in the expansion in powers of $q$ of the value for $x=1 /(a b)$ of the elliptic function $(a b)^{-1 / 2} \phi(u)$. We shall see in a moment that the latter, with $t(u)$, has the periods $u=2 K$ and $2 i K^{\prime}$, and, in many cases, only simple poles. When this is so we can express $\phi(u)$ as a sum of simple theta-quotients with coefficients independent of $u$. For example,

$$
\phi(u)=\sum_{\alpha} A_{\alpha}\left\{\frac{\vartheta_{1}^{\prime}(x-\alpha)}{\vartheta_{1}(x-\alpha)}+\frac{\vartheta_{1}^{\prime}(\alpha)}{\vartheta_{1}(\alpha)}\right\},
$$

the summation being extended to a representative set of poles $x=\alpha$, and the coefficients $A$ being the residues at these poles. When all the $A$ 's are absolute constants, independent of $k$, our problem can be solved, since we have the formula

$$
\frac{\vartheta_{1}^{\prime}(x)}{\vartheta_{1}(x)}=\operatorname{ctn} \pi x+4 \sum_{r=1}^{\infty} \frac{q^{2 r}}{1-q^{2 r}} \sin 2 \pi r x,
$$

with which to form a comparison power series in $q$.

We use the notations of Tannery and Molk, whence

$$
u=2 K x, \tau=i K^{\prime} / K, q=\exp \pi i \tau .
$$

Hence $t(h a u), t(l b u)$ have the periods $x=1, \tau$, and $\phi(u)$ also has these periods. The poles of $t(h a u)$ are the zeros of the denominator $\vartheta_{2}(h a x) \vartheta_{4}(h a x)$, namely

$$
x=\frac{1}{2 a h}\left(m_{1}+m_{2} \tau\right),
$$

where $m_{1}, m_{2}$ are any integers of opposite parities; the zeros of $t($ hau $)$ are given by $(7)$ with $m_{1} \equiv m_{2}(\bmod 2)$. These poles and zeros are simple. Hence, if $p$ is a positive integer, the number $\alpha$ such that

(8) $\alpha=\left(m_{1}+m_{2} \tau\right) /(2 p),\left(m_{1}, m_{2}, p\right)=1, m_{1} \not \equiv m_{2}(\bmod 2)$, is a zero or pole of $t(h a u)$ if and only if $p \mid h a$, and is the former 
or the latter according as $h a / p$ is even or odd. The number of multiples of $p$ in the sequence

$$
a, 2 a, \cdots, \frac{1}{2}(b-1) a,
$$

with an odd quotient for $p$ cannot exceed the number with an even quotient by more than unity. Hence each product $\Pi$ in (2) has only simple poles. If they have poles in common, $\phi(u)$ has a double pole. This occurs, for example, with $p=1$ if $a \equiv b \equiv 3$ $(\bmod 4)$. For no pole $\alpha$ is $\vartheta_{1}(\alpha)=0$.

The poles of $\phi(2 K x)$ in the parallelogram $P \equiv(0,1, \tau, 1+\tau)$ are easily found by the preceding method. If $a=1, b=7$ they are seen at once to be $\rho+\sigma \tau$ and $\sigma+\rho \tau$, where

(9) $(\rho, \sigma)=\left(\frac{1}{2}, 0\right),\left(\frac{1}{4}, 0\right),\left(\frac{1}{4}, \frac{1}{2}\right),\left(\frac{3}{4}, 0\right),\left(\frac{3}{4}, \frac{1}{2}\right),\left(\frac{1}{6}, 0\right),\left(\frac{1}{6}, \frac{1}{3}\right),\left(\frac{1}{6}, \frac{2}{3}\right)$, $\left(\frac{1}{2}, \frac{1}{3}\right),\left(\frac{1}{2}, \frac{2}{3}\right),\left(\frac{5}{6}, 0\right),\left(\frac{5}{6}, \frac{1}{3}\right),\left(\frac{5}{6}, \frac{2}{3}\right)$.

Let us calculate the residue $A$ of $\phi(2 K x)$ at a simple pole $x=\alpha$ of the form (8). If $p$ divides $h a$ with an odd quotient, the limit as $x \rightarrow \alpha$ of

$$
(x-\alpha) \cdot 2 K \cdot t(h a \cdot 2 K x)
$$

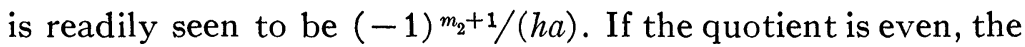
limit as $x \rightarrow \alpha$ of $t(h a \cdot 2 K x) /((x-\alpha) 2 K)$ is simply $h a$. Write $\beta=2 K \alpha$. By (4) and (5) and the preceding remarks, we find

$$
\begin{aligned}
A & =\lim \pi(x-\alpha) \phi(u)=\lim 2 K(x-\alpha) \prod_{l} t(l b u) \prod_{h} t(\text { hau }) \\
& =Q \prod_{l}^{\prime} t(l b \beta) \prod_{h}^{\prime} t(h a \beta),
\end{aligned}
$$

where the primes indicate that the factors for which $p \mid l b$ or $p \mid h a$ are omitted, and $Q$ is the product of the proper numerical

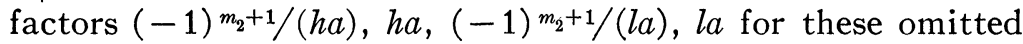
values $h$ and $l$.

Now we readily verify that

$$
\begin{aligned}
t\left(u+i K^{\prime}\right) t(u) & =1=-t(u) t(u+K), \\
t\left(u+2 i K^{\prime}\right) & =t(u)=t(u+2 K), \\
t\left(\frac{1}{2} K\right) & =1, \quad t\left(\frac{1}{2} i K^{\prime}\right)=i .
\end{aligned}
$$

Hence, if $p \beta=(2 m+1) K+2 n i K^{\prime}, m, n$ being integers,

$$
t(r \beta) t(\overline{p-r} \beta)=1=-t(r \beta) t(\overline{p+r} \beta),
$$




$$
t\left(\frac{1}{2} p \beta\right)=(-1)^{m} .
$$

If $p \beta=2 m K+(2 n+1) i K^{\prime}$,

$$
\begin{aligned}
t(r \beta) t(\overline{p-r} \beta) & =-1=-t(r \beta) t(\overline{p+r} \beta), \\
t\left(\frac{1}{2} p \beta\right) & =(-1)^{n} i .
\end{aligned}
$$

Hence in the products $\Pi^{\prime}$ in (10), two factors the sum or difference of whose arguments is $p \beta$ multiplied by an odd integer combine into a constant independent of $k$, and similarly for single factors $t\left(\frac{1}{2} p s \beta\right), s$ odd.

Let $a=1, b \geqq 9$. Consider the pole $\alpha=1 /(b-3)$, whence $p=\frac{1}{2}(b-3), m_{1}=1, m_{2}=0$. Except for a constant factor independent of $k$, the residue $A$ is, by (14) and (15),

$$
t\left(\frac{1}{2}(b-1) 2 K \alpha\right) \text {. }
$$

This actually depends on $k$, since it reduces to 1 if $k=1$, and to $\tan (\pi /(b-3))$ if $k=0$.

Let $b>a>1$, and consider $\alpha=1 /(2 p), p=\frac{1}{2}(b-1) a$. The second factor $\Pi^{\prime}$ in (10) is independent of $k$ as above, but $\Pi_{l} t(l b \beta)$ is seen to depend on $k$, since it has the value 1 if $k=1$, and the value $\Pi_{l} \tan (l b \pi /((b-1) a)) \neq 1$ if $k=0$.

Let $a=1, b=3$. Then $\phi=(2 K / \pi) t(u)$. The poles in $P$ are given by $p=1, m_{1}=1$ or $0, m_{2}=0$ or 1 , and the residues $A$ are respectively -1 and +1 by the rule for forming $Q$.

Let $a=1, b=5$. Then $\phi=(2 K / \pi) t(u) t(2 u)$. Only $p=2$ defines poles; and with it $m_{1}$ is 0 or $2, m_{2}$ is 1 and 3 , and vice-versa. By (15) and (10) the residues $A$ for $m_{1}$ odd are $\frac{1}{2}(-1)^{\left(m_{1}-1\right) / 2}$. By (10) and (17), $A=+\frac{1}{2} i(-1)^{\left(m_{2}-1\right) / 2}$ if $m_{2}$ is odd.

We can easily derive Nazimoff's series for $x^{2}+3 y^{2}$ and $x^{2}+5 y^{2}$.

Let $a=1, b=7, \phi(u)=(2 K / \pi) t(u) t(2 u) t(3 u)$. If $p=1$, each $\Pi^{\prime}$ in (1) is trivially unity, and

$$
A=Q=(-1)^{m_{2}+1} \cdot 2 \cdot(-1)^{m_{2}+1} / 3=\frac{2}{3}
$$

in all cases. If $p=2, Q=(-1)^{m_{2}+1 / 2}$, and $t(\beta) t(3 \beta)=(-1)^{m_{2}+1}$ by (14) and (16). Hence $A=\frac{1}{2}$ in all cases. If $p=3, Q=(-1)^{m_{2}+1 / 3}$, and $t(\beta) t(2 \beta)=(-1)^{m_{2}}$ by (14) and (16). Hence $A=-\frac{1}{3}$.

It follows that this method gives an immediate solution to the problem of finding the formula for $N\left(n=a x^{2}+b y^{2}\right)$ only if $a=1, b=1,3,5,7$. 
Write $x=1 /(a b), \alpha=\rho+\sigma \tau$. On combining (4) and (5), we have to calculate

(18) $\sum_{\alpha} A_{\alpha}\{\sin 2 \pi n \alpha+\sin 2 \pi n(x-\alpha)\}=(1-\cos 2 \pi n x) s_{n}+(\sin 2 \pi n x) c_{n}$, $s_{n}=\sum_{\alpha} A_{\alpha} \sin 2 \pi n \alpha, \quad c_{n}=\sum_{\alpha} A_{\alpha} \cos 2 \pi n \alpha$,

where the summation is extended to the values $\alpha$ in $P$. Write

$$
t_{n}=\sum^{\prime} A_{\alpha} e^{2 n \rho i \pi} q^{2 n \sigma},
$$

where the prime indicates the omission of the values $\alpha=\rho+\sigma \tau$ for which $\sigma=0$. Then

$$
\begin{aligned}
& (20) \\
& \sum_{\alpha} A_{\alpha}\{\operatorname{ctn} \pi \alpha+\operatorname{ctn} \pi(x-\alpha)\}=2 i \sum_{n=1}^{\infty} t_{n}\left(e^{-2 n \pi i x}-1\right) \\
+ & \sum_{\alpha}^{\sigma=0} A_{\alpha}\{\operatorname{ctn} \pi \alpha+\operatorname{ctn} \pi(x-\alpha)\} .
\end{aligned}
$$

Henceforth let $a=1, b=7$. The last sum in (20) is independent of $k$ and must be equal to $7^{1 / 2}$ in view of (1). Since the denominators $p$ of $2 \alpha$ are 1,2 , or 3 the calculation of $s_{n}, c_{n}, t_{n}$ may be done conveniently modulo 6 . For positive integers $r$, we find that

$$
\begin{aligned}
t_{6 r} & =(-1)^{r} q^{6 r}+q^{3 r}+q^{9 r}-q^{2 r}-q^{4 r}-q^{8 r}-q^{10 r}, \\
t_{6 r-5} & =q^{6 r-5}, t_{6 r-4}=q^{3 r-2}+\left\{1+(-1)^{r}\right\} q^{6 r-4}+q^{9 r-6} . \\
t_{6 r-3} & =-q^{2 r-1}+q^{4 r-2}+q^{8 r-4}-q^{10 r-5}, \\
t_{6 r-2} & =q^{3 r-1}+\left\{1+(-1)^{r+1}\right\} q^{6 r-2}+q^{9 r-3}, t_{6 r-1}=q^{6 r-1} .
\end{aligned}
$$

And for any integer $n$,

$$
c_{n}=\frac{1}{2}\left\{t_{n}+t_{-n}\right\}+\gamma_{n}
$$

where $\gamma_{n}$ is the sum of the terms $A e^{2 n \rho i \pi}$ for $\sigma=0$, that is,

$$
\begin{aligned}
& \gamma_{6 r}=(-1)^{r}, \gamma_{6 r-5}=-1, \quad \gamma_{6 r-4}=1+(-1)^{r}, \\
& \gamma_{6 r-3}=0, \quad \gamma_{6 r-2}=1+(-1)^{r+1}, \gamma_{6 r-1}=-1 .
\end{aligned}
$$

Since $t_{n}$ is real for every $n$, and since

$$
s_{n}=-\frac{1}{2} i\left(t_{n}-t_{-n}\right),
$$

$s_{n}$ is purely imaginary. We may drop the imaginary parts, which must annul each other. Hence 
(21)

$$
\begin{aligned}
7^{1 / 2} \sum_{x, y} q^{x^{2}+7 y^{2}}=7^{1 / 2}+ & 2 \sum_{n=1}^{\infty} t_{n} \sin \frac{2 n \pi}{7} \\
& +4 \sum_{n=1}^{\infty} \frac{q^{2 n}}{1-q^{2 n}} c_{n} \sin \frac{2 n \pi}{7}
\end{aligned}
$$

Inserting the values of $t_{n}, c_{n}$ calculated above, we find for the right member of (21) the expression

$$
\begin{aligned}
& 7^{1 / 2}+4 \sum \frac{q^{6 r-5}}{1+q^{6 r-5}} \sin (12 r-10) \frac{\pi}{7} \\
& +4 \sum \frac{q^{6 r-1}}{1+q^{6 r-1}} \sin (12 r-2) \frac{\pi}{7} \\
& +4 \sum\left(\frac{q^{3 r-2}}{1-q^{3 r-2}}+\frac{(-1)^{r} q^{6 r-4}}{1-q^{6 r-4}}\right) \sin (12 r-8) \frac{\pi}{7} \\
& +4 \sum\left(\frac{q^{3 r-1}}{1-q^{3 r-1}}+\frac{(-1)^{r+1} q^{6 r-2}}{1-q^{6 r-2}}\right) \sin (12 r-4) \frac{\pi}{7} \\
& +4 \sum \frac{q^{4 r-2}-q^{2 r-1}}{1+q^{6 r-3}} \sin (12 r-6) \frac{\pi}{7} \\
& +4 \sum \frac{(-1)^{r} q^{6 r}+q^{3 r}-q^{2 r}-q^{4 r}}{1-q^{6 r}} \sin \frac{12 r \pi}{7} .
\end{aligned}
$$

The coefficient of $q^{n}$ in (22) may be calculated as follows. Write $f(m)=2 \sin (2 m \pi / 7)$. Form all factorizations $n=d \delta$ of $n$ into two positive factors and construct the following sums:

$$
\begin{array}{cc}
\sum_{1} f(d)(-1)^{n+1}, & \sum_{2} f(2 d), \\
d \equiv 1,5(\bmod 6) & d \equiv 1,2(\bmod 3) \\
\sum_{3} f(d)(-1)^{d / 2}, & \sum_{4} f(3 d)(-1)^{n}, \\
d \equiv 0,2,4(\bmod 6) & d \equiv 1(\bmod 2), \delta \equiv 1,2(\bmod 3) \\
\sum_{5} f(2 d), & \sum_{6}[-f(3 d)] . \\
d \equiv 0(\bmod 3) & d \equiv 0(\bmod 2) \\
\delta \equiv 1(\bmod 2) & \delta \equiv 1,2(\bmod 3)
\end{array}
$$

Hence we have

(23) $7^{1 / 2} N\left(n=x^{2}+7 y^{2}\right)=2\left(\sum_{1}+\sum_{2}+\cdots+\sum_{6}\right)$. 
If $n=m$ is prime to 6 , this reduces to

(24) $7^{1 / 2} N\left(m=x^{2}+7 y^{2}\right)=2 \sum_{d \mid m}[f(d)+f(2 d)-f(3 d)]$.

We now make use of a (Gauss sum) relation, which may be proved if desired by comparing coefficients of a small power of $q$ in (21):

$$
\sin \frac{2 d \pi}{7}+\sin \frac{4 d \pi}{7}-\sin \frac{6 d \pi}{7}=\frac{1}{2}\left(\frac{d}{7}\right) 7^{1 / 2} .
$$

Hence, if $m$ is prime to 6 ,

$$
N\left(m=x^{2}+7 y^{2}\right)=2 \sum(d \mid 7) .
$$

Making use of (23) for one case we can easily establish by induction the formula

$$
N\left(n=x^{2}+7 y^{2}\right)=2|\alpha-1| \sum(d \mid 7),
$$

where $n=2^{\alpha} m, m$ odd, $\alpha \geqq 0$.

The factor $|\alpha-1|$ for a primary component $2^{\alpha}$ is characteristic of a discriminant $d=4 D, D \equiv 1(\bmod 8)$.

California Institute of Technology 